\section{Predicting immunity}

Two recent studies have used systems biology approaches to identify early gene 'signatures' induced in humans vaccinated with the attenuated yellow fever vaccine YF17D that correlate with, and in some cases predict, the subsequent adaptive immune response.

YF17D, which is one of the most effective vaccines generated so far, is thought to mediate long-lasting protection by inducing neutralizing antibodies, although cytotoxic T-cell responses might also be important. However, a detailed understanding of the early immune response that is induced by YF17D which leads to protection from yellow fever is lacking. The two studies described here used high-throughput technologies, combined with computational modelling in one study, to identify early gene signatures that were induced by YF17D vaccination.

Both studies analysed total peripheral-blood mononuclear cells from different cohorts of human volunteers (who had not been previously vaccinated with YF17D) at various time points following vaccination. Early ( 3 and 7 days postvaccination) effects on gene expression were determined by transcriptional profiling and analysed using several bioinformatics approaches. Many of the genes that were regulated early are involved in the innate immune response, including genes that are associated with Toll-like receptor signalling, the interferon pathway, the antiviral response, the complement pathway and the inflammasome. By visualizing these gene networks, a group of transcription factors, including interferon-regulatory factor 7 , signal transducer and activator of transcription 1 and ETS2, could be identified as key regulators of the early immune response to the YF17D vaccine. In addition, Gaucher et al. showed that YF17D triggers the proliferation and expansion of several immune-cell types (such as macrophages, dendritic cells, natural killer cells and lymphocytes). Together, these data highlight the broad range of innate immune effector mechanisms that are induced by YF17D vaccination.

Although this vaccine is highly effective, the magnitude of the $\mathrm{CD}^{+}$ $\mathrm{T}$-cell responses and antibody titres varied greatly between individuals, but Gaucher et al. found that the $\mathrm{T}$-cell response was of broad epitope specificity and persistent. Querec et al. sought to determine gene signatures that would correlate with and predict the variations in the adaptive immune response; however, none of the genes that had been identified by their transcriptional profiling analyses significantly correlated with the magnitude of the adaptive immune response. Using additional bioinformatics approaches, the authors identified a gene signature that did correlate with the magnitude of antigen-specific CD8 ${ }^{+} \mathrm{T}$-cell responses and antibody titres.

To evaluate the actual predictive capacity of this signature, they determined whether the gene signature could predict the magnitude of the $\mathrm{CD}^{+} \mathrm{T}$-cell or B-cell response in individuals from a second YF17D vaccine trial. They found that several signatures for $\mathrm{CD}^{+} \mathrm{T}$-cell responses from the first trial were predictive with up to $90 \%$ accuracy in the second trial and vice versa. EIF2AK4, which has an important role in the integrated stress response, was repeatedly represented in most of the predictive signatures that were generated, which suggests that this gene could have a central role in mediating the YF17D-induced $\mathrm{CD}^{+} \mathrm{T}$-cell response. Consistent with this, YF17D triggered the integrated stress response in human cells in vitro. In addition, the authors identified a distinct early gene signature that included TNFRSF17 (a receptor for B-cell-activating factor) that predicted the neutralizing antibody titres as late as 90 days following vaccination.

So, these studies provide a detailed description of the transcriptional profile that is induced early after YF17D vaccination and highlight the complexity of the response that is required for the induction of longlasting immune protection. In addition, the magnitude of a protective immune response to YF17D can be predicted early after vaccination using systems biology approaches. These approaches could help to identify early correlates of protection for multiple vaccine candidates and new mechanisms by which vaccines generate protective immune responses.

Olive Leavy

ORIGINAL RESEARCH PAPERS Gaucher, D. et al. Yellow fever vaccine induces integrated multilineage and polyfunctional immune responses.J. Exp. Med. 1 Dec 2008 (doi:10.1084/ jem.20082292)| Querec, T. D. et al. Systems biology approach predicts immunogenicity of the yellow fever vaccine in humans. Nature Immunol. 23 Nov 2008 (doi:10.1038/ni.1688) 